\title{
Intermédialités
}

Histoire et théorie des arts, des lettres et des techniques

Intermediality

History and Theory of the Arts, Literature and Technologies

\section{Raconter : témoigner face au silence de la langue}

\section{Esther Cohen}

Numéro 2, automne 2003

Raconter

Telling

URI : https://id.erudit.org/iderudit/1005457ar

DOI : https://doi.org/10.7202/1005457ar

Aller au sommaire du numéro

Éditeur(s)

Centre de recherche sur l'intermédialité

ISSN

1705-8546 (imprimé)

1920-3136 (numérique)

Découvrir la revue

Citer cet article

Cohen, E. (2003). Raconter : témoigner face au silence de la langue.

Intermédialités / Intermediality, (2), 63-76. https://doi.org/10.7202/1005457ar
Résumé de l'article

Cet article aborde le problème de la crise de la narration qui se produit pendant la Première et Seconde Guerres mondiales, en s'appuyant sur les oeuvres de Primo Levi et Paul Celan, afin de déceler la réponse stratégique que l'on peut donner à cette crise. Leur réponse consiste en un " amenuisement de la langue » qui s'oppose à « l'abréviation du langage " propre au totalitarisme nazi. 


\title{
Raconter : témoigner face au silence de la langue
}

\author{
ESTHER COHEN \\ Traduction de Melina Balcázar et Valérie Juquois
}

Is it possible that the antonym of "forgetting " is not

Remembering but justice?

Yosef Hayim Yerushalmi ${ }^{1}$

Hurbinek n'était rien, c'était un enfant de la mort, un enfant d'Auschwitz. Il ne paraissait pas avoir plus de trois ans, personne ne savait rien de lui, il ne savait pas parler et n'avait pas de nom: ce nom curieux d'Hurbinek lui venait de nous [...]. Hurbinek, qui avait trois ans, qui était peut-être né à Auschwitz et n'avait jamais vu un arbre, Hurbinek, qui avait combattu comme un homme jusqu'au dernier souffle pour entrer dans le monde des hommes dont une puissance bestiale l'avait exclu; Hurbinek le sans-nom dont le minuscule avant-bras portait le tatouage d'Auschwitz; Hurbinek mourut les premiers jours de mars 1945, libre mais non racheté; il témoigne à travers mes paroles ${ }^{2}$.

La moitié de l'art du récit, écrit Walter Benjamin, réside dans la narration d'une histoire libre d'explications, vidée de tout psychologisme imposé ${ }^{3}$. Dans son essai sur le conteur, Benjamin, en 1936, faisait déjà appel à l'austérité de la

1. Yosef Hayim Yerushalmi, «Postscript: Reflections on Forgetting», Zakhor, Jewish History and Jewish Memory, New York, Schocken Books, 1989 [1982], p. 117.

2. Primo Levi, La trêve, trad. Emmanuelle Genevois-Joly, Paris, Éditions Grasset, 2000 [1963], coll. «Les cahiers rouges", p. 25-26. Nous soulignons.

3. Walter Benjamin, «Le conteur. Réflexions sur l'œuvre de Nicolas Leskov », trad. Maurice de Gandillac, Euvres III, Paris, Éditions Gallimard, coll. «Folio Essais », 2000 [1936], p. 117. Désormais les références à cet article seront indiquées par le sigle «LC» suivi de la page et placées entre parenthèses dans le corps du texte. 
chose narrée, à la nécessité de revenir à une tradition où les protagonistes du récit - conteur et auditeur - puissent se livrer une fois encore à la «faculté la plus assurée entre toutes » : raconter des histoires. La Grande Guerre, affirmait-il, est venue mettre en évidence la disparition momentanée de ce métier : «N’avaiton pas constaté, au moment de l'armistice, que les gens revenaient muets du champ de bataille?» (LC, p. 115-116).

Benjamin, avec son regard aigu et prémonitoire, est dans le vrai lorsqu'il entrevoit la fin d'une époque et la chute des facultés de la langue dans la rupture de la civilisation que représenta la Première Guerre mondiale; le silence de ceux qui revenaient muets d'expériences à raconter était déjà un symptôme de la perte d'une mémoire et d'un sens capable de donner un nom à la souffrance, à la bataille, à l'exil et même à la mort. Et en même temps, ce retour sans «gloire» disait déjà ce désenchantement d'une guerre où le soldat avait cessé d'être le héros de l'histoire pour devenir une pièce de plus dans l'engrenage de la masse anonyme, écrasé par la force de la technologie et non par la lutte corps à corps.

Benjamin est suffisamment perceptif pour comprendre que la Grande Guerre marque une rupture, «un bouleversement social et psychologique profond dans lequel il est dès lors commun de voir l'acte fondateur du $\mathrm{XX}^{\mathrm{e}}$ siècle ${ }^{4}$ ». Mais Benjamin, en 1936, trois ans après l'arrivée de Hitler au pouvoir, parle aussi déjà de ce que sera le système totalitaire nazi : le silence de ses héros qui reviennent muets de la guerre se transforme en la grande métaphore de ce que sera la mort, pas seulement cette mort anonyme de la Grande Guerre, mais celle des camps d'extermination nazis, des « cadavres sans mort - à Auschwitz on ne mourait pas, on produisait des cadavres ${ }^{5} »$. Il n'y aura plus de langage capable de rendre compte de ces morts dépouillés de leur propre mort. Et il semble que Benjamin l'ait entrevu dans le mutisme de ces soldats qui, dès 1918, rentrent chez eux sans expériences à relater, sans histoires à raconter. Néanmoins, malgré cette lucidité extraordinaire, la perception de Benjamin ne parviendra pas, oserions-nous dire, à traverser complètement la muraille de l'irracontable: sa clairvoyance s'arrête au bord de l'abîme, car le processus par lequel la langue devient muette n'est encore que latent, et deux ans seulement

4. Enzo Traverso, La violence nazie, Paris, Éditions La fabrique, 2002, p. 23-24.

5. Giorgio Agamben, Quel che resta di Auschwitz, Turin, Bollati Boringhieri, 1998, p. 66. Nous traduisons. 
vont passer pour que le Lager, cet espace concentrationnaire, réduise finalement la langue à un état de mutisme et la brise totalement.

L'authentique aptitude à raconter semblait alors avoir été réduite en cendres, ce que Benjamin lui-même n'était assurément plus prêt à vivre; le Lager est devenu l'endroit où, dans l'entassement et une cohabitation déchirée, l'homme n'a plus trouvé le souffle de la parole de l'autre, mais seulement son silence; étouffée par l'inanition, par cet inane qui est le vide de toute humanité, la parole fut incapable de se raconter les histoires, de se raconter sa propre histoire. Au point que, comme l'a si bien écrit cet autre «annonciateur du feu », Kafka, ce qui est demeuré de cette langue est quelque chose d'à peu près semblable au rire d'Odradek, «le rire de quelqu'un qui n'a pas de poumons, un rire qui a le son du murmure des feuilles mortes ${ }^{6} »$.

Dans ses romans et entretiens, Primo Levi revient sans cesse sur le cauchemar récurrent de ceux qui «habitèrent» les camps: rentrer, raconter et ne pas être écoutés. N'est-ce pas là la prophétie maudite d'Odradek: raconter sans que le conteur puisse se faire entendre, sans l'oxygène nécessaire pour rendre compte de l'inracontable? Ou bien, à l'inverse, ce murmure ne parle-t-il pas, à son tour, de l'atrophie de celui qui ne sait plus poursuivre son récit, de celui qui a perdu sa capacité de mémoire? Car en effet la narration, du moins celle dont parle Benjamin, porte implicitement un savoir ${ }^{7}$, qui passe justement par l'écoute et la mémoire: «L'art de raconter les histoires est toujours l'art de reprendre celles qu'on a entendues, et celui-ci se perd dès lors que les histoires ne sont plus conservées en mémoire. » (LC, p. 118) Jusqu’où, nous demandons-nous, la littérature peut-elle plonger dans son temps et se pénétrer de l'expérience de son époque au point d'être capable de dessiner avec une «exactitude perverse » l'étendue d'une barbarie à venir? Benjamin et Kafka ouvriraient démesurément les yeux et se regarderaient épouvantés, comme l'ange benjaminien de l'histoire, en voyant leurs prémonitions incarnées dans la «catastrophe» du siècle,

6. Franz Kafka, «Le souci du père de famille» [1919], cité dans Harold Bloom, «Kafka», Acta poetica, Instituto de Investigaciones Filológicas, Universidad Nacional Autónoma de México, Mexique, $n^{0 s}$ 9-10, 1990, p. 375. Nous traduisons.

7. «L'art du récit tend à se perdre, parce que l'aspect épique de la vérité, c'est-àdire la sagesse, est en voie de disparition. » (LC, p. 120) Et comme l'écrit Benjamin : «La mémoire est, entre toutes, la faculté la plus nécessaire à l'épopée. » (LC, p. 134) Ce savoir dont il nous parle, cette sagesse, serait directement lié à la capacité de la mémoire, faculté épique et sage par excellence. 
improprement nommée «progrès ». Car le "progrès » est le développement de la technique, même si celle-ci a précisément rendu possible l'existence de ces usines si efficaces à produire des cadavres. Et la langue? Et le conteur? Et le récit? Tout comme Hurbinek-Odradek, il semble qu'ils n'aient pas non plus pu faire face à l'authentique catastrophe; ils devinrent muets, sans pouvoir donner de réponse. La langue, écrit Celan, « est passée à travers et n’a pas eu de mots pour ce qui s'est passé ${ }^{\circledR}$.

Pourtant, et c'est là que réside le mystère de la littérature et de l'art du récit, Celan lui-même écrit: «Nous étions morts et nous pouvions respirer9. » Ainsi, malgré le mutisme imposé par l'anéantissement, il témoigne du vécu; son expérience, ainsi que celle de milliers d'autres, passe par cette phrase claire, austère, «amenuisée». Elle l'habite. Pas de psychologismes, ni d'explications; la parole s'offre à nous toute nue, debout, au milieu du camp, presque juste et austère face à la mort, et en même temps convaincue de cet impossible: respirer dans cette sorte de mort inexplicable et insolite. Le témoignage est là, et c'est ce qui compte. Celan, le poète, nous raconte son histoire et c'est ainsi qu'il résiste à l'agonie et à la destruction, avec ses mots, il défie la barbarie du Lager. Et il le fait de la même manière que l'écriture de Primo Levi est là pour donner vie à l'existence de Hurbinek; car après la leçon concentrationnaire, le témoignage ne peut ni ne doit se borner à rendre compte, il faut qu'il donne vie, qu'il rende l'histoire présente, qu'il l'humanise: le témoin pourrait bien être, aujourd'hui, celui qui résiste, qui s'oppose à l'oubli, c'est-à-dire le conteur que Benjamin recherchait tant et qui semble avoir succombé pendant la Grande Guerre.

Annette Wieviorka, dans son livre L'ère du témoin ${ }^{10}$, soutient que ce n'est qu’à la fin des années 1970 que la figure du témoin surgit de manière consistante en ce qui concerne l'extermination du peuple juif pendant la Deuxième Guerre mondiale. C'est à ce moment-là, insiste-t-elle, que celle-ci acquiert sa force et sa place indiscutable dans l'histoire. D'autres chercheurs, comme Enzo Traverso, situent l'émergence de cette figure du témoin en 1961, année du procès Eichmann en Israël, où l'on considère presque le témoignage comme

8. Paul Celan, Obras completas, trad. José Luis Reyna Palazón, Valladolid, Trotta, 1999, p. 497. Nous traduisons.

9. Paul Celan, Obras completas, p. 57.

10. Annette Wieviorka, L'ère du témoin, Paris, Éditions Hachette littératures, coll. «Pluriel », 2002. 
un processus thérapeutique au cours duquel, pour la première fois, «celui qui a réussi à revenir » a pu dire sa parole, raconter son histoire ${ }^{11}$. Cela ne veut bien sûr pas dire qu'avant ces dates on n'ait pas parlé de l'univers concentrationnaire, mais on peut dire que celui-ci n'avait jusqu'alors pas occupé de place déterminante dans la réponse collective au phénomène qui a marqué d'une manière si incontestable l'expérience du $\mathrm{xx}^{\mathrm{e}}$ siècle.

Or, Primo Levi, et en cela sa voix se situe de manière intempestive face à l'«ère du témoin ", écrit lorsque personne encore ne veut rien savoir de l'expérience des camps; 1947, année où paraît Si c'est un homme, est un moment où, même pour les victimes qui subirent le Lager, ce que l'on cherche, c'est à se réintégrer à la société après l'expérience dévastatrice de l'extermination. Peu nombreuses sont les oreilles disposées à écouter l'horreur de la barbarie, de la même façon que peu nombreuses furent les victimes qui décidèrent d'écrire leur propre témoignage. Jorge Semprún lui-même a choisi, dit-il, la vie et non l'écriture, et ce n'est que quarante ans après qu'il se décide à écrire son récit intime, L'écriture ou la vie ${ }^{12}$.

C'est pourquoi la parole de Primo Levi est, à plus d'un titre, un acte intrépide, épique, presque de résistance, lucide, austère, conscient malgré cette courte distance, et qui essaie à tout prix de récupérer, par la narration, le choc de l'expérience vécue. Levi reprend ce que Benjamin appelle la dimension orale de la narration; d'où la reprise constante de ce récit dans un de ses nombreux entretiens :

Je suis revenu du Lager avec une charge narrative qui était même pathologique. Je me souviens très bien de certains voyages en train en 1945, à peine rentré, quand je parcourais l'Italie pour retrouver, pour reconstruire ma situation professionnelle: je cherchais du travail. Et dans le train, je me souviens avoir raconté mes affaires au premier venu. À ce propos, j’ai cité le vieux marin de Coleridge, qui raconte son histoire à des gens se rendant à un mariage et qui se moquent de lui. Eh bien, je faisais exactement la même chose ${ }^{13}$.

Primo Levi, avant d'écrire, commence par raconter selon la bonne vieille manière du conteur benjaminien. Il est conscient qu'il faut raconter, que c'est

11. Enzo Traverso, La historia desgarrada. Ensayo sobre Auschwitz y los intelectuales, trad. David Chiner, Barcelone, Herder, 2001.

12. Jorge Semprún, L'écriture ou la vie, Paris, Éditions Gallimard, 1994.

13. Primo Levi, Entrevistas y conversaciones, Barcelone, Éditions Península, 1998, p. 130. Nous traduisons. 
là un devoir. Et cela parce que, pour lui, l'écrivain, il ne s'agit pas tant de raconter «son» histoire personnelle comme un acte disons thérapeutique, que de rendre justice à ceux qu'il appelle «les naufragés », de donner la parole à ces morts qui ne reposeront en paix pas même après la «victoire ", à moins qu'une voix capable de leur donner une sépulture humaine ne se fasse entendre.

Mais témoigner d'un monde et d'une langue fracturés exige, après Auschwitz, non seulement une résistance, mais une justice qui va bien au-delà de la loi. Être témoin, dans de telles circonstances, cesse d'être un acte d'héroïsme. On n'est plus un martyr, c'est-à-dire, en grec, un témoin, car on ne meurt pas pour témoigner de la foi, on ne paie plus le témoignage de sa vie. Mais il ne s'agit pas non plus du témoin judiciaire de la culture latine, dans lequel une sorte de discrimination est en jeu dans la langue, puisque lui seul, le testis, qui, comme son nom l'indique, a des testicules et en même temps est témoin, peut exercer cette fonction. L'héroïcité aussi bien que la force de la loi restent en dehors du témoignage concentrationnaire. Et il ne s'agit pas non plus du témoin qui nous est familier, capable de témoigner de n’importe quel événement, aussi banal fût-il. Il faudrait nommer autrement celui qui, comme Primo Levi, fait un acte de justice quotidien qui passe nécessairement par l'écriture, par cette narration de l'expérience qui semble avoir disparu avec la guerre de 1914-1918.

En racontant la vie des camps par écrit et dans cette prose explicite, sans détours, elle aussi amenuisée par l'expérience, l'écrivain devient une sorte d'ange benjaminien de l'histoire; mais à la différence de celui-ci, celui-là non seulement contemple, épouvanté, les ruines du passé, mais il raconte, et en ce sens agit, transformant son récit en action dans la meilleure tradition du conteur: dans sa narration, il y a toujours un «conseil » pour celui qui écoute, une leçon de vie (LC, p. 119-120). La voix de Primo Levi n’est pas celle du héros: quel héroïsme pourrait-il y avoir dans la description d'un sous-monde où le persécuté et le persécuteur, chacun à sa manière, ont perdu leur visage? Ce n'est pas non plus celle du juge implacable qui réclame justice ou qui crie vengeance. Porter témoignage à travers la parole écrite c'est, pour Levi, rendre justice, en exigeant que la singularité la plus absolue, par définition incommunicable, soit néanmoins l'occasion de tirer une leçon d'histoire. Porter témoignage, c'est retrouver la parole, c'est, écrit Benjamin, «raconter sa vie et sa dignité » (LC, p. 150), permettant ainsi que, dans la figure du conteur, «le juste se rencontre lui-même» (LC, p. 151). Car la justice est étroitement liée à cette capacité de raconter des histoires, ici l'histoire des camps, avec sa violence et son trouble. Bref, ce qui est juste irait de pair avec l'écriture de la barbarie. 
Raconter est donc, dans ce contexte, un acte de justice qui interpelle une «communauté» plutôt que l'individu dans sa singularité. Témoigner, et dans une large mesure le récit de la deuxième moitié du siècle est marqué par le témoignage (il suffit de penser à la quantité de récits aussi bien écrits que filmés sur ce qui constitua l'événement de la Deuxième Guerre mondiale), c'est prendre la parole de l'autre, parler à sa place et, à la différence du héros ou du témoin judiciaire, celui qui écrit prête sa voix à ceux qui ont, en leur temps, été dans l'impossibilité de parler ou empêchés de se faire entendre. Mais surtout, celui qui témoigne récupère une dimension politique et sociale du récit que le cours du temps avait diminuée. Car, écrit Levi,

[...] c'est justement parce que le Lager est une monstrueuse machine à fabriquer des bêtes, que nous ne devons pas devenir des bêtes; puisque même ici il est possible de survivre, nous devons vouloir survivre, pour raconter, pour témoigner; et pour vivre, il est important de sauver au moins l'ossature, la charpente, la forme de la civilisation ${ }^{14}$.

Ils nous enlèveront jusqu'à notre nom: et si nous voulons le conserver, nous devrons trouver en nous la force nécessaire pour que derrière ce nom, quelque chose de nous, de ce que nous étions, subsiste ${ }^{15}$.

C'est que dans le nom se concentre ce qu'il y a de plus intime et de «racontable» dans l'être humain. Nous sommes ce que notre nom a accumulé pendant toute une vie, le nom est ce «signe volumineux» dont parle Roland Barthes ${ }^{16}$, chargé d'une épaisseur pleine de sens ambigus et contradictoires, un signe vaste et spacieux qui loge la vie comme la mort; «le nom est la frontière qui sépare le territoire du sens et le désert du délire ${ }^{17}$ ». Il faut donc s'accrocher à lui car, pourrait dire Levi, d'où peut-on mourir si l'on n'a pas un nom d'où l'on puisse le faire, un lieu où la mort puisse être certifiée? Un numéro tatoué ne répond pas du sens d'une vie et d'une mort, un numéro ne parle que de

14. Primo Levi, Si c'est un homme, trad. Martine Schruoffeneger, Paris, Éditions Julliard, 1987 [1947], p. 42

15. Primo Levi, Si c'est un homme, p. 26.

16. Roland Barthes, «Proust et les noms», To honour Roman Jakobson, La Haye, Éditions Mouton, 1967, repris dans Le degré zéro de l'écriture suivi de Nouveaux essais critiques, Paris, Éditions du Seuil, coll. «Points», 1972 [1953], p. 125.

17. Voir Esther Cohen, El silencio del nombre, interpretacion y pensamiento judio, Barcelone, Mexique, Éditions Rubi, Anthropos, Fundación Eduardo Cohen, 1999, p. 43. Nous traduisons. 
l'abîme du délire. Les morts d'Auschwitz ne sont donc pas des morts authentiques mais autre chose, car comment l'homme peut-il mourir s'il a été dépouillé de cette maison intime que nous habitons tous et que l'on appelle nom propre? Et si nous en sommes dépouillés, ce qui reste, c'est cette chose que Levi décrit comme la figure du «musulman ${ }^{18} »$, cette sorte de mort vivant incapable déjà de se souvenir de l'existence même d'un nom, de la permanence de sa demeure la plus intime et la plus humaine. «Ce sont eux, les Muselmänner», écrit Levi,

les damnés, le nerf du camp; eux, la masse anonyme, continuellement renouvelée et toujours identique, des non-hommes en qui l'étincelle divine s'est éteinte, et qui marchent et peinent en silence, trop vides déjà pour souffrir vraiment. On hésite à les appeler des vivants: on hésite à appeler mort une mort qu'ils ne craignent pas parce qu'ils sont trop épuisés pour la comprendre. Ils peuplent ma mémoire de leur présence sans visage, et si je pouvais résumer tout le mal de notre temps en une seule image, je choisirais cette vision qui m’est familière: un homme décharné, le front courbé et les épaules voûtées, dont le visage et les yeux ne reflètent nulle trace de pensée ${ }^{19}$.

À leur égard, les naufragés des camps, il s'agit maintenant, pour Levi, de raconter les histoires, non par un acte de pure volonté, mais par un exercice $d u$ devoir: on doit survivre parce qu'on doit raconter. On doit lutter contre le projet d'extermination accompagné du plus lourd des silences, c'est-à-dire contre le projet formulé par Himmler en 1943:

18. Multiples sont les interprétations données pour expliquer pourquoi le terme «musulman» a occupé cette place dans les camps. Certains soutiennent que c'est à cause du degré de dénutrition, des basses températures et de la position de leurs corps qui, vus de loin, pouvaient donner l'impression d'Arabes en train de prier. Mais d'après Agamben, l'explication la plus plausible renvoie à la signification du terme arabe muslim, qui signifie «celui qui se soumet inconditionnellement à la volonté de Dieu » et qui se trouve à l'origine de la légende du supposé fatalisme islamique, si répandue dans les cultures européennes depuis le Moyen Âge (Giorgio Agamben, Ce qui reste d'Auschwitz, p. 46). Il y a d'autres explications, comme celle que l'Encyclopédie judaïque donne du terme Muselmann: «Utilisé surtout à Auschwitz, le terme semble dériver de la position typique de ces déportés, c'est-à-dire prostrés à terre, les jambes repliées à la façon orientale, le visage rigide comme un masque.» (Cité dans Giorgio Agamben, Ce qui reste d'Auschwitz, p. 47)

19. Primo Levi, Si c'est un homme, p. 96-97. 
Il a fallu prendre la grave décision de faire disparaître ce peuple de la terre [...]. Il ne subsistera que des restes de population juive qui auront trouvé abri quelque part. [...] Vous êtes maintenant au courant, et vous garderez tout cela pour vous. Bien plus tard, on pourra peut-être poser la question de savoir s'il faut en dire plus au peuple allemand. Je crois qu'il a mieux valu que nous - nous tous - prenions cela sur nos épaules pour notre peuple, que nous en prenions la responsabilité (la responsabilité d'un acte et non d'une idée) et que nous emportions notre secret avec nous dans la tombe ${ }^{20}$

Il faudra alors combattre ce secret voué à la tombe, briser ce projet de faire disparaître toute trace de l'extermination. Comme le rapporte Levi dans Les naufragés et les rescapés, les S.S. s'amusaient, cyniques, à avertir les prisonniers:

Quelle que soit l'issue de cette guerre, la guerre contre vous, nous l'avons gagnée; aucun de vous ne restera pour raconter, et même si l'un de vous parvenait à en réchapper, le monde ne le croirait pas. Peut-être y aura-t-il des soupçons, des discussions, des recherches des historiens, mais il ne pourra y avoir aucune certitude, car les preuves seront détruites avec vous. Et si une preuve arrive à subsister, si l'un de vous parvient à survivre, les gens diront que les faits que vous racontez sont trop monstrueux pour être crus : ils diront que ce sont des exagérations de la propagande alliée, et c'est nous qu'ils croiront, nous qui nierons tout, pas vous. L'histoire du Lager, c'est nous qui l'écrirons ${ }^{21}$.

C'est pourquoi l'histoire du Lager doit être écrite par ceux qui, comme Levi, font exister la vie d'un Hurbinek par leur écriture, une vie plus juste du fait même qu'elle est racontée par l'écrivain. Et en effet, il ne s'agit pas seulement, concrètement, de la vie de cet enfant, mais de celle de l'humanité souffrante tout entière, des naufragés et des musulmans du camp; et Levi, lorsqu'il parle de son expérience, parle simultanément de notre réalité potentielle à tous, même si nous n’avons certes pas été «des habitants des camps». Car la barbarie, à partir de l'expérience concentrationnaire du $\mathrm{xx}^{\mathrm{e}}$ siècle, est déjà intégrée à notre processus de civilisation. Elle fait partie de notre héritage. Hurbinek et Levi sont des personnages de notre temps, ils font partie de l'histoire la plus intime et la plus déchirée de notre $\mathrm{Xx}^{\mathrm{e}}$ siècle, et de ce fait le récit de l'écrivain aussi bien que la non-vie d'un sans-nom appelé Hurbinek interpellent non seulement l'individu mais la communauté, non pas la bonne conscience du

20. Heinrich Himmler, discours prononcé devant les Reichsleiter et les Gauleiter à Posen, le 6 octobre 1943, cité dans Annette Wieviorka, L'ère du témoin, p. 19.

21. Primo Levi, Los hundidos y los salvados, trad. Pilar Gómez Bedate, Barcelone, Éditions Muchnik, 2000 [1986], p. 11. Nous soulignons, nous traduisons. 
sujet mais la justice quotidienne du monde où nous vivons. L'expérience des camps nous interpelle tous dans la mesure où, comme l'écrivait Hannah Arendt en 1946, dans la «solution finale» il n'était pas uniquement question de tuer pour des raisons humaines: un système d'extermination avait été créé dont la fin ultime consistait à «supprimer le concept d'être humain ${ }^{22}$ ». Sous cette perspective le témoignage se constitue, dans l'espace de la narration, sur la terre où la justice se transforme de jour en jour, où elle s'actualise quotidiennement et où, de nouveau avec Benjamin, «le juste se rencontre lui-même». Rendre justice en racontant ce qui est arrivé, c'est, en ce sens, empêcher que le concept d'être humain, ou d'espèce humaine, comme l'appelle Robert Antelme ${ }^{23}$, disparaisse de notre vocabulaire, de notre « paysage » conceptuel, politique et social.

Claude Lanzmann, dans son film Shoah (1985), tourné pendant onze ans, a insisté plus d'une fois sur le fait que, à aucun moment, il n'a prétendu faire un documentaire historique. Il n'y a pas, dans les neuf heures que dure le film, une image d'archives, pas un seul mort, incinéré ou gazé, pas une seule scène de violence. Cependant, dans les camps vides que la caméra parcourt lentement, en silence ou dans le sifflement des trains qui traversent les camps déserts, au présent, comme si l'événement qu'il raconte n'appartenait pas au passé, Shoah se constitue (se construit) en un témoignage qui interpelle implacablement la justice, cette justice qui est au-delà de la reconstitution des faits ou du châtiment des coupables. Lanzmann, dans ce langage mis entre guillemets, imperturbable, de la caméra qui parcourt avec la même sérénité apparente les lieux, maintenant vides, de l'horreur, ainsi que dans les voix de ses interviewés, parfois dans des endroits splendides comme les plages de Tel Aviv, se propose d'exercer une justice qui pour beaucoup s'apparente à celle de Levi et, avec lui, à celle que Benjamin exige de tout conteur.

L'effet dramatique des témoignages, tant chez les survivants que chez les nazis, ne surgit pas, à quelques exceptions près, de l'exploitation de l'image déchirée du témoin, ni même d'un excès d'information; au contraire, ce sont les images «amenuisées» des trains et des camps, vides et plongés dans le silence, comme écoutant au loin les voix de ceux qui les ont un jour peuplés,

22. Hannah Arendt, Karl Jaspers, Briefwechsel [1926-1969], Munich, Éditions Piper, 1985, p. 106, cité dans Enzo Traverso, La historia desgarrada, p. 86. Nous traduisons.

23. Robert Antelme, L'espèce humaine, Paris, Éditions Gallimard, 1957. 
qui témoignent de l'horreur, faisant ainsi un acte de justice. Lanzmann suit soigneusement les récits de ses témoins, mais il sait que la vérité ne passe pas par la seule information qu'ils possèdent; il faut qu'une autre instance vienne tirer le langage (y compris le langage cinématographique) du mal où il est tombé. Il ne s'agit pas de communiquer, comme l'écrit Derrida dans Force de loi: «De ce point de vue, le nazisme a vraiment été la figure la plus notable de la violence médiatique et de l'exploitation politique des techniques du langage de la communication, du langage industriel et du langage de l'industrie ${ }^{24}$. » Il s'agit plutôt, justement, de déconstruire ce langage de la communication et de le faire passer, en l'amenuisant, par le filtre du racontable. Et ici, le racontable serait synonyme de ce qui est juste. Car la justice ne réside pas dans la divulgation des archives mais dans les interstices que celles-ci laissent à découvert.

Nous avons insisté sur l'expression «amenuiser la langue», pour nous référer tant à la poésie de Celan qu'à la prose de Primo Levi ou au langage cinématographique de Lanzmann. Mais il faudrait préciser: amenuiser n’est pas abréger. Un abîme sépare ces deux verbes: le premier nous conduit au conteur épuré et «juste», l'autre nous renvoie le visage du nazisme. Ce qui peut être abrégé, c'est précisément ce qui ne peut pas être raconté, ce qui passe par la mutilation de la mémoire; ce qui peut être abrégé est l'organisation et la technique. Victor Klemperer, philologue allemand, a consacré une grande partie de sa vie à étudier la langue du nazisme: «Depuis le premier jour de la guerre et jusqu'à la chute du Troisième Reich, tout ce qui est hérö̈que sur terre, sur mer et dans le ciel, porte l'uniforme militaire ${ }^{25}$.» Et la langue n'y échappe pas, elle aussi prend les traits du Führer: elle devient rigide, dure, imperméable, sa voix se transforme en un cri qui ordonne, qui appelle le peuple, les camarades du Peuple, les ennemis du Peuple, les voisins du PEuple, etc., ce peuple toujours en majuscules et qui n'a rien à voir avec ses gens ni leurs histoire. Puisque le Troisième Reich, et avec lui, sa langue, n’est fait, écrit Klemperer, que de solennité, on pourrait dire qu'il souffre d'un manque de quotidienneté et que sa maladie est mortelle... ${ }^{26}$ La langue du Troisième Reich n'amenuise pas, elle abrège; ce faisant, elle annule toute possibilité de remé-

24. Jacques Derrida, Force de loi. Le fondement mystique de l'autorité, Paris, Éditions Galilée, coll. «La philosophie en effet», 1994, p. 143.

25. Victor Klemperer, LTI, la langue du Troisième Reich, trad. Elisabeth Guilot, Paris, Albin Michel, coll. «Bibliothèque idées», 1996 [1947], p. 27.

26. Victor Klemperer, LTI, la langue du Troisième Reich, p. 68. 
moration et fige toute parole en une formule apprise qui n'a rien à voir avec l'authentique mémoire racontable. Éternel, fanatique, solennel, promesse fanatique, fanatique profession de foi, Peuple, Organisation: dans ces termes, le nazisme coagule la langue allemande, expulsant d'elle toute capacité à raconter l'histoire, et avec elle, toute humanité. Le nazisme est, du début à la fin, une paralysie de la mémoire, et sa langue, une langue tout en majuscules qui a perdu le don d'être narrativisée, c'est-à-dire amenée sur le terrain de la lettre minuscule, du quotidien et du témoignable.

Walter Benjamin, dans ses thèses «Sur le concept d'histoire » écrites en 1940, et déjà sous le coup de ce que voulait dire pour lui le pacte germanosoviétique, écrivait:

Faire œuvre d'historien ne signifie pas savoir «comment les choses se sont réellement passées ». Cela signifie s'emparer d'un souvenir, tel qu'il surgit à l'instant du danger. Il s'agit pour le matérialisme historique de retenir l'image du passé qui s'offre inopinément au sujet historique à l'instant du danger. Ce danger menace aussi bien les contenus de la tradition que ses destinataires. Il est le même pour les uns et pour les autres, et consiste pour eux à se faire l'instrument de la classe dominante. À chaque époque, il faut chercher à arracher de nouveau la tradition au conformisme qui est sur le point de la subjuguer. Car le messie ne vient pas seulement comme rédempteur, il vient comme vainqueur de l'antéchrist. Le don d'attiser dans le passé l'étincelle de l'espérance n’appartient qu'à l'historiographe intimement persuadé que, si l'ennemi triomphe, même les morts ne seront pas en sûreté. Et cet ennemi n'a pas fini de triompher ${ }^{27}$.

Connaître l'histoire, reconnaître notre passé ne signifie donc pas le connâ̂tre «tel qu'il a réellement été», c'est-à-dire dans sa conception historique positiviste, mais le saisir dans cet instant de danger où l'histoire resplendit un moment, presque comme les cendres chaudes d'un feu sur le point de mourir et qui rougeoient encore un instant avant de s'éteindre définitivement. Comprendre l'histoire des camps n'est pas accepter passivement l'histoire officielle, toujours racontée par les vainqueurs, même s'il ne s'agit pas, dans ce cas, des vainqueurs nazis. Il s'agit plutôt de «brosser l'histoire à rebrousse-poil ${ }^{28}$ », autre expression benjaminienne utilisée dans la thèse VII et qui vient réfuter toute lecture linéaire de l'histoire; il s'agit de la lire et de pénétrer en elle en cet

27. Walter Benjamin, «Sur le concept d'histoire», Euvres III, Paris, Éditions Gallimard, coll. «Folio essais», 2000 [1940], p. 431. Nous soulignons.

28. Walter Benjamin, «Sur le concept d'histoire», Euvres III, p. 433. 
instant foudroyant de danger, de la lire à contre-courant. Comme Levi, il faut s'approprier des souvenirs, tel celui de Hurbinek, qu'il retrouve dans ces moments de danger où le nom, et avec lui l'homme, perdaient leur visage, c'està-dire leur dignité. Primo Levi, à travers son écriture et la sensibilité au danger que sa propre expérience lui a donnée - il le disait souvent lui-même dans ses entretiens, Auschwitz fut pour lui une autre université, où il apprit des choses qu'il n'aurait apprises nulle part ailleurs - , fut capable de s'approprier une parcelle de l'histoire, et ce faisant, il réussit à lui rendre justice.

En ce sens, l'écrivain Primo Levi devient une sorte d'historien benjaminien lorsqu'il sait, et son écriture le montre, que même les morts ne seront pas à l'abri de l'ennemi si celui-ci triomphe. Et il ne s'agit pas d'une menace qui se présenterait «nécessairement sous la forme primitive et grossière de la restauration monarchique des Stuart maltraitant les ossements de Cromwell, mais par la falsification ou l'oubli de leurs combats ${ }^{29}$ ». Les morts sont bien morts, mais il faut leur donner un autre type de sépulture, leur donner dans l'histoire une place où leur vie prenne sens, au moins ce sens que peut lui donner un conteur comme celui visé par Benjamin. Or, l'ennemi, comme le note ce dernier, n’a pas fini de triompher. Et à l'époque où il écrit les Thèses, en 1940, c'est déjà «le minuit du siècle» en Europe. Les victoires de l'ennemi, pour reprendre à nouveau Löwy, «étaient monumentales: défaite de l'Espagne républicaine, pacte germano-soviétique, occupation de l'Europe par le III ${ }^{\mathrm{e}}$ Reich.» Cependant, «malgré sa vocation de Cassandre et son pessimisme radical, Benjamin ne pouvait pas prévoir Auschwitz ${ }^{30}$ ».

C'est pourquoi il faut donner un nom et une voix à ceux qui, de leur vivant, ne les ont pas eus. Il faut que l'histoire du Lager ne soit pas écrite par les vainqueurs; que cette histoire soit écrite par les autres, au nom des vaincus, des naufragés, est un devoir. Il faut permettre aux morts de reposer enfin en paix. C'est pourquoi nous devons être vigilants. Nous vivons des temps obscurs, écrivait Celan, et les ombres construisent pas à pas leurs propres espaces : c'est pourquoi, et pour paraphraser Calvino, il faudra voir si de fait nous vivons dans les ombres de l'enfer ${ }^{31}$ - et Auschwitz fut l'enfer - , voir qui et ce qui dans cet

29. Michael Löwy, Walter Benjamin: Avertissement d'incendie. Une lecture des thèses "Sur le concept d'histoire», Paris, Presses universitaires de France, 2001, p. 51.

30. Michael Löwy, Walter Benjamin: Avertissement d'incendie, p. 53.

31. Italo Calvino, Las ciudades invisibles, trad. Aurora Bernárdez, Barcelone, Éditions Minotauro, 1985 [1972]. 
univers n'est pas l'enfer, n'est pas obscurité ni barbarie, pour lui donner un espace et le faire durer. Hurbinek, dit Primo Levi, n’a pas survécu au camp, il est mort avant d'être racheté; mais grâce à l'écriture de Levi, il a quitté ce lieu des ténèbres et de la barbarie, il a retrouvé dans les mots de l'écrivain son humanité perdue, et obtenu du récit de sa vie une sépulture humaine. 